\title{
IN SITU SUSTAINED EULERIAN OBSERVATORIES
}

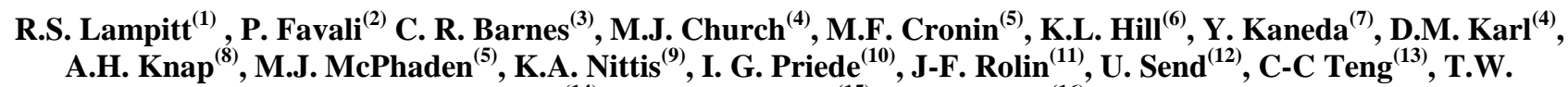
Trull $^{(14)}$, D.W.R. Wallace ${ }^{(15)}$, R.A. Weller ${ }^{(16)}$

${ }^{(1)}$ National Oceanography Centre, Southampton, SO14 3ZH, UK. Email: R.Lampitt@noc.soton.ac.ukmailto:

${ }^{(2)}$ Istituto Nazionale di Geofisica e Vulcanologia, Italy.Email: paolo.favali@ingv.it

${ }^{(3)}$ NEPTUNE Canada, University of Victoria, P.O. Box 1700, STN CSC, Victoria, BC V8W 2Y2, Canada

Email: crbarnes@uvic.ca

(4) Department of Oceanography, 1000 Pope Rd. MSB 629, University of Hawaii, Honolulu, HI 96822 USA. Email: mjchurch@hawaii.edu and dkarl@hawaii.edu

${ }^{(5)}$ NOAA PMEL, 7600 Sand Point Way NE, Seattle, WA 98115 USA.

Email: Meghan.F.Cronin@noaa.gov and michael.j.mcphaden@noaa.gov

(6) Integrated Marine Observing System, University of Tasmania, Private Bag 110, Hobart 7000, Australia. Email: Katy.Hill@imos.org.au

(7) Japan Agency for Marine-Earth Science and Technology (JAMSTEC), DONET, 2-15 Natsuhima-cho, Yokosuka 2370061, Japan Email: kaneday@jamstec.go.jp

${ }^{(8)}$ Bermuda Institute of Ocean Sciences, Inc., 17 Biological Lane, Ferry Reach, GE01, Bermuda. Email: tony.knap@bios.edu

(9) Hellenic Centre for Marine Research, 46.7 km Athens-Sounio Ave., PO Box 712, Anavyssos, Attica, GR-190 13, Greece.Email: knittis@ath.hcmr.gr

${ }^{(10)}$ University of Aberdeen, Oceanlab, Main Street, Newburgh, AB41 6AA, UK. Email: i.g.priede@abdn.ac.ukmailto:

(II) IFREMER, Centre de Brest, BP 70, 29280 Plouzané, France.Email: jrolin@ifremer.fr

${ }^{(12)}$ Scripps Institution of Oceanography, Mail Code 0230, University of California, San Diego, La Jolla, CA 92093 0230,USA. Email: usend@ucsd.edu

${ }^{(13)}$ NOAA National Data Buoy Center, Stennis Space Center, MS 39529, U.S.A. Email: chung-chu.teng@noaa.gov

${ }^{(14)}$ Antarctic CRC, Univ. of Tasmania, GPO Box 252-80, Hobart, 7001, Australia. Email:Tom.Trull@utas.edu.au

${ }^{(15)}$ Leibniz-Institut für Meereswissenschaften (IFM-GEOMAR) Marine Biogeochemie-Chemische Ozeanographie Düsternbrooker Weg 20, 24105 Kiel, Germany.Email:dwallace@ifm-geomar.de

${ }^{(16)}$ Woods Hole Oceanographic Institution, 266 Woods Hole Rd., MS\# 29, Woods Hole, Ma. 02543, USA. Email: rweller@whoi.edu

\begin{abstract}
In order to gain a better understanding of the interactions of processes and properties of the earth system and how these are changing with time, it is essential that there is a sustained stream of high quality data on the marine environment. This must extend from its surface to the underlying seabed and use a matrix of interlinked platform types, each with specific advantages. Included in this matrix is the global array of fixed point or Eulerian observatories which have several unique capabilities. These include the ability to collect samples (water, biota and particles), to support sensors which have a high demand for space or power, to make observations in locations beyond the reach of satellites, gliders and floats and to observe and sample the seafloor. Considerable progress has been made in the operability of these observatories over the past decade and some, such as the Global Tropical Moored Buoy Array, make physical and meteorological observations that are well integrated. There has been considerable
\end{abstract}

progress in sensor development, platform design, and in the principles and protocols required for data management. There is however a significant requirement now to interlink observations on biogeochemistry within the global Eulerian array and between Eulerian observatories and the other observing systems in the matrix. Large scale computational models closely coupled to the various observational approaches are required for much of this work and this challenge is being addressed by a number of groups. For this to be achieved there must be a change in mind-set of many of the funding organisations so that the financial resources are sustained. This is essential in order to prevent breakage of data streams and loss of the skill base of staff at the end of every funding round. There are examples of this change in approach in the USA (OOI (Observatories Initiative)) and in Canada (NEPTUNE (North East Pacific Time-series Undersea Networked Experiments)) but elsewhere in the world, short term funding is usually the normal mechanism 
which is expected to address long term environmental questions of major societal relevance.

\section{INTRODUCTION}

Observations of the processes and properties of any natural system are often frustrated by temporal variability, which cannot be adequately characterised. When combined with spatial variability this has been a major impediment to our understanding of system structure and function and, in particular, the determination of natural or anthropogenic changes with time. In the oceanic water column, the situation is significantly more difficult than in terrestrial systems because of its greater volume and larger vertical scales and the fact that spatial variability is frequently not fixed geographically. The properties of ocean gyres for instance change with time but also sometimes advect horizontally in their entirety at rates up to $15 \mathrm{~km} / \mathrm{d}$ [1]. Satellite remote sensing can provide insights into the scales and magnitude of spatial and temporal variability of surface waters [2] but provide limited information about properties or processes occurring below the sea surface or their scales. For some types of satellite sensors such as those for ocean colour (e.g. SeaWiFS (Sea-viewing Wide Field-of-view Sensor)), even the presence of clouds or atmospheric pollution dramatically reduces data acquisition. Temporal variability occurring at or below the seafloor can be easier in some ways to explore due to constant geography and, for some purposes; these environments provide a very attractive setting, which integrates in both space and time.

Eulerian observatories are defined as platforms that are fixed with respect to the earth crust at one location and which incorporate sensors for making in situ measurements of the properties of the surrounding water or sediment or the processes occurring there. They may also collect samples of water, biota or solids and incorporate a variety of sensors from different disciplines. This has the advantage of shared logistics (the platforms can be expensive to construct, deploy and maintain) and possible interdisciplinary observations and collaborations such as on the relationship between physical, chemical, geological and biological properties. Today all such observatories are unmanned although in the past the weather ships functioned as Eulerian observatories and provided invaluable insights. Station Mike for instance in the Norwegian sea provided almost daily data from 1947 leading to the longest record of deep water hydrography in the world and a clear record of a significant temperature increase of about $0.1^{\circ} \mathrm{C}$ at $2000 \mathrm{~m}$ from about 1986 until the ship was withdrawn in 2009 [3]. In addition, at some of the sites such as at the Hawaii Ocean Time-series observatory and many of the coastal and shelf locations, a large proportion of the data is derived from repeated ship occupations.
This paper reviews Eulerian ocean observatories presenting the rationale for the approach, the current level of development and a vision for the future. Other ocean observing systems and platforms are reviewed elsewhere [2, 4, 5, 6, 7, 8 and 9]. There is no doubt that as part of an integrated and diverse range of observing systems, Eulerian observatories provide an invaluable and unique insight into the ways in which the oceans are changing with time.

\subsection{Rationale behind Eulerian observatories}

The justification and rationale for the various ways of observing the oceans has been forcefully made in the introduction to this volume and in the conference statement and the variety of societal and scientific needs require no further reiteration. Eulerian platforms form a crucial component in this diverse array due to their unique capabilities not available to other observing systems (see below). During the past two decades, there have been a large number of workshops, meetings and publications discussing the need for Eulerian time series observations. Many reports have been written as part of the process to establish the Ocean Observatories Initiative (OOI) in the USA or the EuroGOOS (European Global Ocean Observing System) and ESONET (European Seas Observatory NETwork) programmes in Europe:

http://www.oceanleadership.org/programs-andpartnerships/ocean-observing/ooi/documents-andpublications/;

http://www.eurogoos.org/content/content.asp?menu=00 50000000000000000

These frequently involved contributions from the international community and it is unnecessary to repeat the many valid arguments, which have been published beyond the following summary.

In order to explore and understand the time changing properties of the oceanic environment, sustained observations at a sufficiently high frequency are essential. Such observations provide the means to examine complex interrelations between processes and properties at various scales from minutes to decades. The time and space scales of a wide variety of environmental processes have been elegantly presented by [10] and demonstrate the scales of observation which are required if insights are to be obtained (Fig. 1). To take surface tides as an example of the reasons for high frequency, it is clearly inadequate to make observations with a temporal resolution of 12 hours (the tidal period is in most regions 12.42 hours). In this particular instance and probably many others, such poor temporal resolution does not simply prevent an accurate measurement of temporal variability but would provide a clear but entirely incorrect assessment due to aliasing. If an environmental signal is less pronounced and there is no a priori understanding of the likely periodicity, 
aliasing is a particular danger and observation should be adopted at the highest frequency possible. In addition to the dangers of aliasing, short term or episodic events may well be missed if the sampling frequency is too low. It is widely believed that these short-term events such as blooms, storms and surges have a disproportionate effect on the functioning of all marine environments and without high frequency sampling, it is impossible to understand their effects.

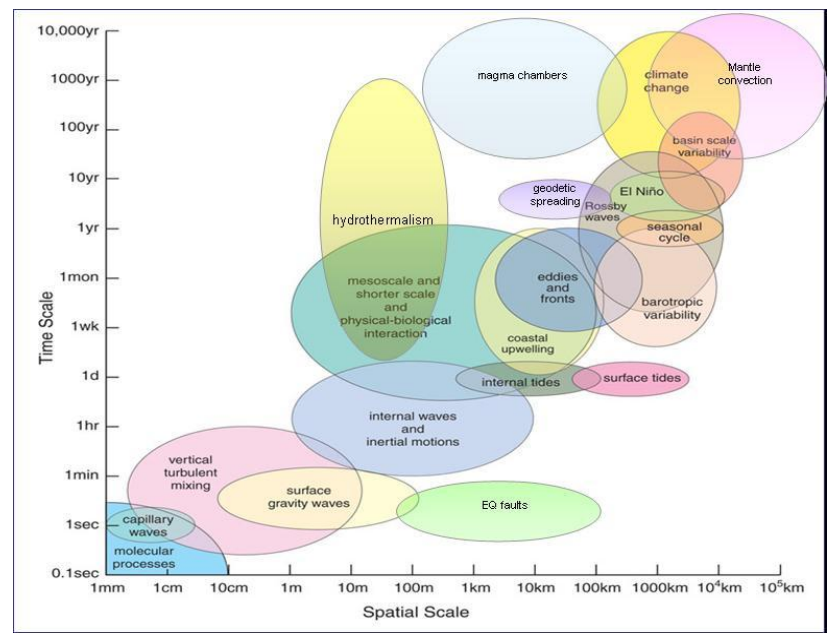

Figure 1. Time and space overlapping scales of major ocean and earth processes (redrawn from work of $D$. Chelton and L.Beranzoli, and from [11].

There are two different but complementary rationales for sustained time series observations. The first is to understand the time varying properties of different components of a system in order to gain insights into the interactions between these components. Consequently, a greater understanding can be obtained about system function. An example might be the relationship between changes in the concentration of surface nutrients and phytoplankton and that of the downward flux of particles in the deep ocean [12].

The second rationale is to determine whether and how the system is changing on a longer time scale, which may or may not be related to anthropogenic activity. This could be considered as "monitoring", an activity, which is essential if we are to detect longer term, changes in the global environment. Both of these objectives demand consistent observations, which are of sufficiently high frequency to avoid aliasing and for these to be sustained over prolonged periods of time. With regard to currently observed anthropogenic changes, such as in ocean $\mathrm{pH}$, there is clearly no predetermined end date for observations and the community of scientists, policy makers and funding agencies need to adopt a mind-set that such observations must become an integral and constant feature in the same way in which highways are maintained and the wages of doctors are paid. Both these rationales for time series observations demand continuing investment in both equipment and people.

An important facet of these two reasons is that the observatories provide well-calibrated, in situ observations. Models, either statistical or numerical, as well as remote sensing products and climatologies or data syntheses may have errors, biases, and limited space/time resolution. The sustained Eulerian sites are essential for capturing actual in-situ variability and for their ability to associate known uncertainties (from calibrations) with their time series. At the same time, the sustained time series sites are essential as validation and verification benchmarks for models, for remote sensing, and for synthesized fields of data.

During the past decade the scientific community has become progressively more aware of the need for time series measurements and many governments and funding bodies support sustained observing structures whether they use ships of opportunity (e.g. CPR (Continuous Plankton Recorder) and CALCOFI (California Cooperative Oceanic Fisheries Investigations)) [4, 6, 13, 14 and 15], repeat transects by research vessels such as the Repeat Hydrography Lines [16] (http://go-ship.org, ), research vessels or deployed instruments for benthic observations (e.g. [17 and 18]) gliders and floats, or water column Eulerian observations [19]. In addition, research vessels with skilled personnel are required to make the measurements of some properties of the ocean or rates of processes within it, which cannot be measured autonomously.

There have been some major technological developments over the past decade both in the capabilities of floats and gliders [9, 20 and 21] and in the sensors, which they carry [22 and 23]. However, although the size and power requirements of sensors and samplers are becoming smaller, many are still not appropriate for mobile autonomous platforms when used for prolonged periods because of their space, calibration and power demands. As a consequence, and particularly for sampling of the water, particulate material or the biota, fixed point Eulerian observatories are still an essential part of the observational tool kit. For them, limitation of electrical power is much less severe whether using batteries, solar panels or cables from shore and the size and weight of the sensor or sampler is also rarely a limiting factor. In the benthic and sub-seafloor environments, the observations are almost by definition Eulerian although benthic crawlers have been used for some applications in order to obtain material or make rate measurements from a location which has not been affected by previous sampling (e.g. [24] NEPTUNE Canada (North East Pacific Time-series Undersea Networked Experiments)). To summarise, Eulerian observatories are the essential observational 
platform under circumstances where the demands are for:

1. high power, which is often the case when multiple sensors, crawlers or profilers are needed;

2. real-time sub-surface data supply;

3. repeated site-specific sample collection (Water, biota, particles);

4. deep ocean location (below the maximum depth of floats and gliders), or other locations not well measured by drifters, floats, ships or satellites;

5. seafloor and sub-seafloor processes (i.e. interactions of geo-, bio- and hydro-sphere).

6. high frequency, unaliased data, such as is required for a reference data set.

In terms of data supply systems, these multi-sensor platforms can have different configurations: (a) in stand-alone or delayed mode or (b) with acoustic, satellite or cabled capabilities. Several fixed observatories currently have the characteristic (or aspiration) of being real-time, multidisciplinary, interactive and, in addition, some cover a range of different environments from the upper surface of the ocean to the seabed beneath e.g. the Porcupine Abyssal Plain (PAP) observatory in the northeast Atlantic

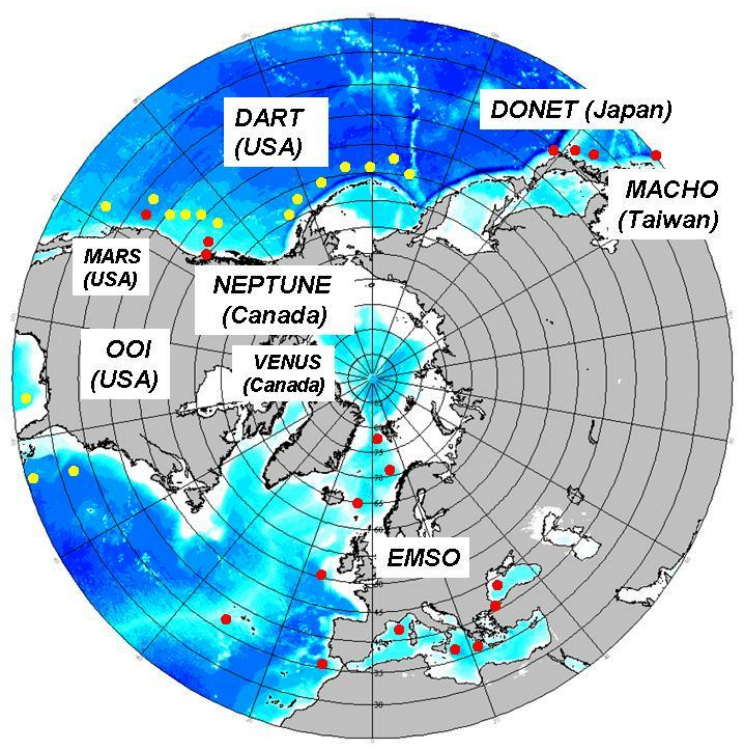

Figure 2. Geographical distribution of the major seafloor observatories.

(http://www.noc.soton.ac.uk/pap/ [25]), and the MARS (Monterey Accelerated Research System), VENUS (Victoria Experimental Network Under the Sea) and NEPTUNE Canada cabled observatories (Fig. 2) http://www.neptunecanada.ca/. Real-time data supply is an advantage for three distinct reasons. The first and most important has its origins in the fact that the oceans are a hostile environment and losses of equipment are not uncommon. Real-time data supply to shore ensures data security and even if data storage devices become flooded or the entire observatory is lost, previously collected data are still secure. The second reason is that for some variables there is a requirement for an operational system which can either be used for computational models such as for ecosystem management or for early warning of hazards such as of earthquake or tsunami. The third reason is that it provides the opportunity for human interaction with the observatory which may be to change the sampling regime in response to new data or to effect repairs as a result of system malfunction (remotely or on site).

As discussed in the accompanying community white papers (e.g. [17, 18, and 19]), Eulerian observatories address a variety of issues but some of the most important are:

1. The role of the ocean in climate change

2. Turbulent mixing and biophysical interactions

3. Ecosystem dynamics and biodiversity

4. Fluids and life in the ocean crust

5. The dynamics of the lithosphere and imaging of the Earth's interior

They can and do cover many research priorities:

- Biogeochemistry (e.g. elemental cycling, including most notably the global carbon cycle through both physical and biological processes);

- Marine ecology (e.g. distribution and abundance of sea life, ocean productivity, biodiversity, ecosystem function, living resources, and climate feedbacks);

- Physical oceanography (e.g. water mass characterisation, water column processes, thermodynamics, ice cover, climatology, and impacts on climate change);

- Geosciences (e.g. transfer from Earth's interior to the crust, hydrosphere and biosphere, fluid flow and gas seepage through sediments and gas hydrate, nonliving resources, sediment transfer to deep-sea and climate change);

- Geo-hazards (e.g. earthquake and tsunami hazard, volcanic hazard, slope instability and failure).

\section{CURRENT STATE}

Within the last two decades, it has been appreciated by the community at large that if Eulerian observatories are going to fulfil their potential, they must be integrated into networks. These networks need to share common standards both in terms of their sensors, interfaces and metrology but also and most importantly in terms of 
data management [26]. There are three general categories of such observatories: (a) Shelf and Coastal water column; (b) Open ocean water column and (c) Seafloor and sub-seafloor. In the majority of cases, but not all, the observatories would be categorised as either "research" or "pre-operational". Especially for biological and chemical variables, there is a significant challenge ahead to develop sensors which have the necessary durability, reliability and sensitivity and then for the data to be standardised so that it can be compared with data from other designs of sensor measuring the same variable. Although the rate of progress has been rapid over the past decade, at the time of writing most of the biological and chemical sensors could not be described as operational. Exceptions to this are salinity, oxygen and fluorescence.

\subsection{Shelf and coastal water column}

One of the more difficult categories in which to develop coherent and common goals and protocols has been in the shelf and coastal waters. The reasons for this is that these observatories have greatest relevance to national interests, are more focused on local goods and services and hence the responsible agents have less of a global perspective. Nevertheless there has been some significant progress [27, 28 and 29] and some, such as those in the Baltic Sea (http://www.iowarnemuende.de/marnet-en.html) and the Integrated Marine Observing System around Australia (IMOS) [30] (http://www.imos.org.au/ ), are well integrated. In the former case, the observatory can be considered as an operational system providing a management tool for coastal systems. IMOS is still at an early stage but a good example of the integration of approaches is found in the fact that the arrays are deployed with other platforms such as HF radar and gliders to utilize the combined strength of these platforms. From a European perspective, the European Marine Strategy Framework Directive will be a significant driver to develop a more integrated approach. This involves evaluation of ecological status and establishment of a list of key parameters with an associated monitoring policy.

\subsection{Open ocean water column}

Over the last decade there has been a concerted effort by OceanSITES (OCEAN Sustained Interdisciplinary Time series Environment observation System) to integrate the open ocean water column observatories (www.OceanSITES.org [19]). OceanSITES is a worldwide network of nearly 100 long-term, multidisciplinary deepwater reference stations measuring dozens of variables and monitoring the full depth of the ocean from air-sea interactions down to 5,000 meters ( $97 \%$ of the sea surface). In addition, the Global Tropical Moored Buoy Array (GTMBA) (Fig. 3) provides a wealth of data on the physics and meteorology of the tropical regions of the major oceans (see below). Since 1999, the international OceanSITES team has promoted the value of such observing systems and has provided the driving force to integrate diverse observatories and to harmonise methods of working. Although the issue of sustained funding for such observatories remains a major challenge, there has been considerable progress in achieving international agreement on many of the key
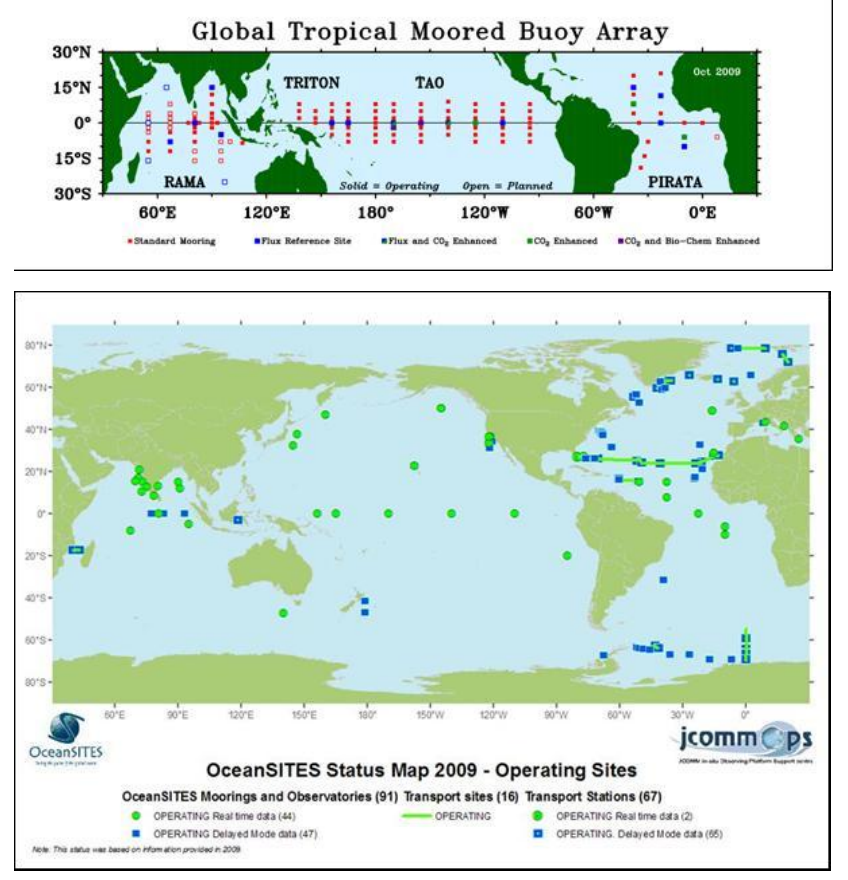

Figure 3. Top: Locations of observatories of upper ocean physics and meteorology integrated under the Global Tropical Moored Buoy Array (GTMBA).

Bottom: Location of OceanSITES observatories in addition to those of the GTMBA.

issues such as data management and protocols. There is an underlying fundamental principle that the data should enter the public domain without restriction and that this must occur as soon as they are available. In the case of real-time data, this implies a timescale of a few hours and for delayed mode data (stored in situ and retrieved along with the instrument), a few weeks is the maximum delay. The observatories were established with a variety of objectives and with diverse funding streams making comparisons between them of common variables very challenging. However, it is intended that within the next few years there will be a common set of variables, which can be directly compared across the entire ocean system. These will be variables of relevance to climate and will include $p \mathrm{CO}_{2},\left[\mathrm{O}_{2}\right]$, upper water column physical structure and light distribution and are an extension of the Essential Climate Variables (ECVs) proposed by the Global Climate Observing System (GCOS). GCOS has defined a set of 44 ECVs 
that should be monitored with respect to climate change in accordance with the requirements of the UN Framework Convention on Climate Change (UNFCCC) and the Kyoto Protocol. However, at present the focus is most strongly on variables derived from satellites and does not therefore take advantage of data currently measured by ocean Eulerian observatories.

Figure 3 shows the locations of the observatories from which it can be seen that there are still some large gaps in coverage and some of these are in regions of the oceans, which are of considerable significance from an earth system perspective. The expectation is that during the coming years observatories will be deployed in these gaps, an issue which will be addressed by OceanSITES (see below).

Servicing of Eulerian observatories is a significant consideration in the open ocean environment where transit times from the shore can be several days or weeks and where weather conditions may be harsh and unpredictable. The consequence of this is that the resources available may prevent servicing more frequently than once per year. There are exceptions to this such as the Hawaii Ocean Time-series, (HOT) and the Bermuda Atlantic Time-series Study (BATS) where there has been sustained commitment to monthly occupations of selected ocean sites by research vessels with associated moorings, floats, and gliders. For other open ocean observatories, however, durability and reliability of both the sensors and platforms whether they be benthic landers or water column moorings are key factors affecting the viability of open ocean observatories. Many of the more remote locations are very poorly sampled and until observatories can be deployed with improved reliability for one or several years, such regions are bound to be under-sampled. Funding bodies are sensitive to applications to deploy observatories in remote regions because of the associated long-term financial and logistic commitments during times when projections of their research funding often have large uncertainties. With better sensors and platforms and funding streams, which are distinct from those of research, the situation will be significantly improved.

One of the best examples of an integrated sustained open ocean network is the Global Tropical Moored Buoy Array (GTMBA) (Fig. 3) [31] which is a component of OceanSITES. This provides real time data on the upper ocean physics and meteorology across the tropical Indian, Pacific and Atlantic oceans using over 100 moorings with surface buoys. Data from the arrays are used to advance understanding of processes that govern climate dynamics originating in the tropics such as the El Niño/Southern Oscillation, the Indian Ocean Dipole, tropical Atlantic climate variability, the mean seasonal cycle, the intraseasonal Madden-Julian Oscillation as well as the decadal variability and trends in these. The data are also used to develop, initialize, and validate forecast models for advance warning of weather and climate events that have socio-economic consequences. TAO/TRITON (Tropical Atmosphere Ocean/Triangle Trans-Ocean Buoy Network) in the Pacific is the oldest of the arrays, with origins dating back 30 years [32] but the Indian and Atlantic arrays are expanding in number and all are expected to deploy a variety of additional sensors in the future including those addressing biogeochemistry.

While most open ocean observatories can be considered as "pre-operational", there are several examples of ones, which are entirely research based and may not be directly related to climate issues. A good example of this is the use of passive acoustic systems to examine the behaviour of marine mammals such as the European LIDO (LIstening to the Deep Ocean) one of the demonstration missions currently running within the EC project ESONET-NoE http://www.listentothedeep.com/. Similarly, photographic techniques have been used to examine temporal variability of so-called "marine snow" particles (those greater than $0.5 \mathrm{~mm}$ diameter) which are responsible for the majority of the downward flux of material in the ocean [33].

\subsection{Seafloor and sub-seafloor}

Many international projects have been planning permanent networks of observatories placed on the seafloor or within boreholes [17]. Figure 2 shows the geographical distribution of the major cabled or acoustic-linked projects although there are significant numbers of additional seafloor observatories, which store data exclusively in situ. A global network of seafloor observatories will help to understand the ocean, and the complex physical, biological, chemical, and geological systems operating within it.

Seafloor observatories offer the opportunity to study multiple, interrelated scientific processes over varying time scales, as is the case for water column observatories. Episodic processes include volcanic eruptions, earthquakes, tsunamis, and biological, chemical and physical impacts of storm events and those biogeochemical changes driven by environmental perturbations at the sea surface above. An example comes from the NEPTUNE Canada project where it has been found that surface tides can be quantitatively related to changes in sediment pore pressures and infaunal behaviour and to behaviour of many benthic organisms. The dynamics of the lithosphere, which has been monitored during the past centuries by fixed geodetic stations on land, is a good example of global and long-term processes to be observed also on the seafloor. Having a network of seafloor observatories represents a new direction in ocean science research, and requires a major investment of human and economic resources over many decades. This 
environment encompasses the major interface between ocean biology/biogeochemistry and that of the geological sciences and is therefore one where multidisciplinary science is most pronounced (see below). From a biogeochemical perspective, benthic observatories record data, which can be considered to integrate water column processes in time and over a large surface area of the ocean. Consequently, they may be less subject to spatial variability than in the overlying water column and thus have a significant role in the ocean observing system.

The linkages between the three categories described above have not in the past been well developed. The PAP observatory in the Northeast Atlantic is probably the only one in the world, which has a strong focus on the seafloor, the midwater and the upper water column [25]. However, NEPTUNE Canada, operating from 2009, and OOI, being installed by 2014 , use the new generation of cabled observatories, which are able to provide abundant power and high bandwidth real-time communications from a combination of seafloor instrument platforms as well as connecting to instruments within boreholes and on both moorings and vertical profilers through the water column. These can also serve as subsea power sources for docking AUVs. NEPTUNE Canada has five node observatories with over 130 instruments (several hundred sensors) in water depths ranging from 100-2660 meters connected by an $800 \mathrm{~km}$ backbone cable and so can be characterized as a network of five Eulerian observatories gathering realtime 4-D data (60 terabytes/year) over a large area.

\section{A VISION FOR THE FUTURE}

Agreement is universal in the earth system community that if our understanding of the oceans and underlying seabed is to be enhanced, it will be essential for us to have a sustained stream of high quality time series data. These data must be of a wide variety of system properties some of which will be variables in the properties recorded such as temperature or $p \mathrm{CO}_{2}$ (state variables) while others will record the rates of processes such as primary production or pore water flow (rate variables). Much of this endeavour will involve simultaneous measurements of diverse variables and this may be in an interdisciplinary framework defined as one in which the variables have the potential to affect one another. An example may be in the rates and characteristics of primary production in the surface waters, which will affect the ecology of large benthic fauna several thousand meters beneath [34]. Interdisciplinary studies clearly address large-scale science questions as well as having the logistical advantages of deployment of equipment in remote locations. Logistic advantage can also be gained from multidisciplinary observations in which the variables being measured are unlikely to affect each other but can take place at the same location. An example may be contemporaneous measurements of $\mathrm{PCO}_{2}$ near the surface and pore fluid migration in the sediment, a process which is affected by earthquakes. In these cases of multidisciplinary observations, considerably more discussion is required in order to reach appropriate compromises as the optimal locations of study for the various disciplines may be very different. Nevertheless, the advantages in terms of reduced logistical and infrastructure costs could be substantial.

As stated above, there are various platforms, which can be used for time series measurements of the oceanic environment each of them with advantages and shortcomings. With the increasing reliability of gliders and floats combined with decreasing size and power requirements of sensors, the role of Eulerian platforms becomes very specific as described above under "Rationale". They now make a unique and highly prized contribution to the overall observing capability. The seafloor and sub-seafloor environment will be environments where fixed platforms will also continue to be the technique of choice for time series studies; the new generation of cabled observatories allows connected instrumentation in, on and above the seafloor and networked over large areas, thus providing a remarkable integration of ocean data from a wide range of environments and detecting short and long term events and processes.

One of the most serious impediments to the development of a coherent and integrated observational capability of the oceans is that much of the data from the different observational approaches (Eulerian obs., gliders, satellites etc) is not at present integrated into a modelling framework. This is particularly the case for biological and biogeochemical data sets. For physical measurements, model based analysis and reanalysis systems are well established and that make use of a range of in situ and satellite data sets for climate, navigation, military applications, oil spill response, coastal zone management, etc. (e.g., [35, 36 and 37]). The challenge now is to extend these systems to other types of data. This is not a trivial task and demands firstly that the observational data from all sources are consistently supplied and that this is performed at an appropriate frequency (e.g. every few hours). Secondly, the data must be managed in a consistent way across the approaches with, for instance, quality control processes carried out uniformly and metadata adequately presented [26 and 38]. Thirdly, the modelling community must play a key role in harmonising the community of observationalists. From the specific perspective of Eulerian observations, the modelling community must in addition play a pivotal role in identifying where additional observatories are required. The strategy is that the output from models will be used to rank the various regions of the ocean according to a 
combination of 1: how well they are currently observed; and 2: how essential are the data for various specified purposes. Clearly, this is to be carried out in the context of the list of essential climate variables and when completed there will be a priority list of regions in which observatories should be deployed or existing ones enhanced.

The overall vision is to develop a global network of multidisciplinary and interdisciplinary sustained Eulerian observatories, which is intimately linked to all of the other observational approaches (gliders, floats, satellites, etc). The process of integration must be continued so that comparisons between data from the various observing systems are simple and reliable. From the perspective of Eulerian observations there are a number of regional programmes, which are working together such as EuroSITES, ESONET, EMSO (European Multidisciplinary Seafloor Observatory) NEPTUNE Canada and OOI and these make major contributions to international networks such as OceanSITES. In general, the principles of data management have been established and agreement is at an advanced stage about "best practice".

The vision outlined above is of course dependent on sustained funding and this is perhaps the greatest threat to the development of an integrated observing system. Apart from NEPTUNE Canada and OOI, which have secured large and prolonged national support, a major problem with almost all other observatories is that funding is available for only a few years at a time and frequently from research budgets, which generally have short time perspectives. European funding is designed to enhance integration but again the timescale is short (3-5 years) and the progress made during one project or funding cycle is frequently lost at the end of the cycle. This is a thoroughly bad method to invest in such crucially important observational capacity and at present, some significant reorganisation is required in order that budgets available for instance for satellite remote sensing and for data management are made available for in situ observation.

On a more positive note, there is optimism in the community that the existing infrastructure provides an excellent basis on which to build. Integration within the Eulerian observatory network is progressing well, sensors are being developed and improved at a high rate, computational models are evolving rapidly, and multidisciplinary teams are learning to accommodate the massive flows of real-time data. The future network will have a much larger range of sensors, measurements and samplers such that the environmental descriptions will be far more insightful and will enable much deeper understanding of system function and changes over time in response to natural variability and anthropogenic perturbation.
The vision for the future of Eulerian observatories is dependent on continuing progress in certain areas and more substantial stimulus in other areas.

In summary:

\section{Areas where progress is currently good}

1. Sensor development

2. Platform design

3. Data management principles and protocols

4. Computational basin-scale models

\section{Areas in which additional stimulus is required}

1. Integration of biological and biogeochemical data from Eulerian observatories and other observing capability into a unified modelling framework.

2. Objective determination of which regions of the oceans require Eulerian observatories to be deployed or existing ones to be upgraded or redeployed.

3. Separation of "research funding" from operational funding required for long term sustained observations. Eulerian observatories should be funded as a small number of sustained large-scale research infrastructures.

\section{REFERENCES}

1. Chelton, D.B., Schlax, M.G., Samelson, R.M., \& de Szoeke, R.A. (2007). Global observations of large oceanic eddies, Geophys. Res. Lett., 34, L15606, doi:10.1029/2007GL030812.

2. Sathyendranath, S. \& Co-Authors (2010). "ChloroGIN: Use of Satellite and In Situ Data in Support of EcosystemBased Management of Marine Resources" in these proceedings (Vol. 2), doi:10.5270/OceanObs09.cwp.75.

3. Dickson B, Osterhus S (2007). One hundred years in the Norwegian Sea. Norsk Geografisk Tidsskrift-Norwegian Journal of Geography, 61(2): 56-75

4. Goni, G. \& Co-Authors (2010). "The Ship of Opportunity Program" in these proceedings (Vol. 2), doi:10.5270/OceanObs09.cwp.35.

5. Gunn, J., Rogers, A. and Urban, E., (2010). "Observation of Ocean Biology on a Global Scale: Implementing BioGOOS?" in these proceedings (Vol. 1), doi:10.5270/OceanObs09.pp.20

6. Reid, P. \& Co-Authors (2010). "A Global Continuous Plankton Recorder Programme" in these proceedings (Vol. 2), doi:10.5270/OceanObs09.cwp.73 .

7. Roemmich, D. \& Co-Authors (2010). "Integrating the Ocean Observing System: Mobile Platforms" in these proceedings (Vol. 1), doi:10.5270/OceanObs09.pp.33.

8. Send, U. \& Co-Authors (2010). "Towards an Integrated Observing System: In Situ Observations" in these proceedings (Vol. 1), doi:10.5270/OceanObs09.pp.35. 
9. Testor, P. \& Co-Authors (2010). "Gliders as a Component of Future Observing Systems" in these proceedings (Vol. 2), doi:10.5270/OceanObs09.cwp.89.

10. Chang, G.C. \& Dickey, T.D. (2001). Optical and physical variability on time-scales from minutes to the seasonal cycle on the New England shelf: July 1996 - June 1997, J. Geophys. Res., 106, 9435-9453.

11. Lampitt, R. \& Co-Authors (2010). "Long-term Biogeochemical Time-Series from the Porcupine Abyssal Plain Deep Ocean Observatory, North East Atlantic". In these proceedings (Annex).

12. Lampitt, R. and Larkin K.E. and the EuroSITES Consortium. "EuroSITES European Network of Deep Ocean Observatories" In these proceedings (Annex).

13. Garzoli, S. \& Co-Authors (2010). "Progressing Towards Global Sustained Deep Ocean Observations" in these proceedings (Vol. 2), doi:10.5270/OceanObs09.cwp.34.

14. Kent, E. \& Co-Authors (2010). "The Voluntary Observing Ship (VOS) Scheme" in these proceedings (Vol. 2), doi:10.5270/OceanObs09.cwp.48.

15. Koslow, J., Goericke, R., McClatchie, S., Vetter, R. and Rogers-Bennett, L., (2010). "The California Cooperative Oceanic Fisheries Investigations (CalCOFI): The Continuing Evolution and Contributions of a 60-Year Ocean Observation Program" in these proceedings (Vol. 2), doi:10.5270/OceanObs09.cwp.49.

16. Hood, M. \& Co-Authors (2010). "Ship-Based Repeat Hydrography: A Strategy for a Sustained Global Program." in these proceedings (Vol. 2), doi:10.5270/OceanObs09.cwp.44.

17. Favali, P. \& Co-Authors (2010). "Seafloor Observatory Science" in these proceedings (Vol. 2), doi:10.5270/OceanObs09.cwp.28.

18. Larkin, K. \& Co-Authors (2010). "Benthic Biology TimeSeries in the Deep Sea: Indicators of Change" in these proceedings (Vol. 2), doi:10.5270/OceanObs09.cwp.52.

19. Send, U. \& Co-Authors (2010). "OceanSITES" in these proceedings (Vol. 2), doi:10.5270/OceanObs09.cwp.79.

20. Claustre, H. \& Co-Authors (2010). "Bio-Optical Profiling Floats as New Observational Tools for Biogeochemical and Ecosystem Studies: Potential Synergies with Ocean Color Remote Sensing." in these proceedings (Vol. 2), doi:10.5270/OceanObs09.cwp.17.

21. Freeland, H. \& Co-Authors (2010)."Argo - A Decade of Progress" in these proceedings (Vol. 2), doi:10.5270/OceanObs09.cwp.32.

22. Adornato, L. \& Co-Authors (2010). "In Situ Nutrient Sensors for Ocean Observing Systems" in these proceedings (Vol. 2), doi:10.5270/OceanObs09.cwp.01.

23. Brasseur, L., Tamburri, M. and Pluedemann, A., (2010). "Sensor Needs and Readiness Levels for Ocean Observing: An Example from the Ocean Observatories Initiative (OOI)" in these proceedings (Vol. 2), doi:10.5270/OceanObs09.cwp.10.
24. McGill, P.R., Sherman, A.D., Hobson, B.W., Henthorn, R.G. \& Smith, Jr. K.L. (2009). Initial deployments of the Rover, an autonomous bottom-transecting instrument platform. J. Ocean. Tech. 4, 9-26. (18 pages)

25. Lampitt, R.S., Billett, D.S.M. and Martin, A.P. (2010) Water column and seabed studies at the PAP sustained observatory in the Northeast Atlantic. Eds. Deep Sea Research Part II: Topical Studies in Oceanography, 57, (15).

26. Pouliquen, S., Schmid, C., Wong, A., Guinehut, S. and Belbeoch, M., (2010). "Argo Data Management" in these proceedings (Vol. 2), doi:10.5270/OceanObs09.cwp.70

27. Malone, T. \& Co-Authors (2010). "Building a Global System of Systems for the Coastal Ocean: A Strategic Action Plan for Implementing the Coastal Module of GOOS" in these proceedings (Vol. 2), doi:10.5270/OceanObs09.cwp.59.

28. UNESCO (2003). The Integrated Strategic Design Plan for the Coastal Ocean Observations Module of the Global Ocean Observing System. GOOS Report No.125, pp. 190.

29. UNESCO (2005). An Implementation Strategy for the Coastal Module of the Global Ocean Observing System. GOOS Report No.148, pp. 141.

30. Hill K, Moltmann T., Meyers G., Proctor R. "The Australian Integrated Marine Observing System (IMOS)". In these proceedings (Annex).

31. McPhaden, M. \& Co-Authors (2010). "The Global Tropical Moored Buoy Array" in these proceedings (Vol. 2), doi:10.5270/OceanObs09.cwp.61

32. McPhaden, M. J., A. J. Busalacchi, and D. L. T. Anderson, 2010b: A TOGA Retrospective. Oceanography, 23, 86103.

33. Lampitt R.S., Hillier W.R. and Challenor P.G., (1993) Seasonal and diel variation in the Open Ocean concentration of Marine Snow Aggregates. Nature 362:737-739.

34. Wigham, B.D., Hudson, I.R., Billett, D.S.M. \& Wolff, G.A. (2003). Is long-term change in the abyssal Northeast Atlantic driven by qualitative changes in export flux? Evidence from selective feeding in deep-sea holothurians. Progress in Oceanography, 59, (4), 409441. doi:10.1016/j.pocean.2003.11.003.

35. Bahurel, P. \& Co-Authors (2010). "Ocean Monitoring and Forecasting Core Services, the European MyOcean Example" in these proceedings (Vol. 1), doi:10.5270/OceanObs09.pp.02

36. Rienecker, M. \& Co-Authors (2010). "Synthesis and Assimilation Systems - Essential Adjuncts to the Global Ocean Observing System" in these proceedings (Vol. 1), doi:10.5270/OceanObs09.pp.31

37. Xue, Y. \& Co-Authors (2010). "Ocean State Estimation for Global Ocean Monitoring: ENSO and Beyond ENSO" in these proceedings (Vol. 2), doi:10.5270/OceanObs09.cwp.95. 
38. Blower, J. \& Co-Authors (2010). "Ocean Data Dissemination: New Challenges for Data Integration" in these proceedings (Vol. 1), doi:10.5270/OceanObs09.pp.05. 